\title{
SEMICLOSED OPERATORS IN HILBERT SPACE
}

\author{
WILLIAM E. KAUFMAN
}

\begin{abstract}
In a Hilbert space $H$, an operator $C$ is semiclosed provided that there exists a bounded operator $B$ on $H$, with range the domain of $C$, such that $C B$ is bounded. The family of all such operators in $H$ is the smallest family containing all closed operators and itself closed under any one of the following: (1) sums, (2) products, (3) strong limits on domains of closed operators. In fact, every algebraic combination of closed operators in $H$ is the sum of two closed one-to-one operators with the same domain and closed ranges.
\end{abstract}

Introduction. Suppose that $(H,\langle\cdot, \cdot\rangle)$ is a complete complex inner product space (i.e., a Hilbert space) and let $(H \times H,\langle\cdot, \cdot\rangle \sim)$ denote the usual product space. The statement that $S$ is a semiclosed subspace of $H$ means that $S$ is a linear (but not necessarily closed) subspace of $H$ for which there is an inner product $\langle\cdot, \cdot\rangle^{\prime}$ such that $\left(S,\langle\cdot, \cdot\rangle^{\prime}\right)$ is complete and continuously included in $H$, in the sense that there is a nonnegative number $b$ such that $\langle x, x\rangle\left\langle b^{2}\langle x, x\rangle^{\prime}\right.$ for all $x$ in $S$. Now, an operator in $H$ is understood to be a linear function from a linear subspace of $H$ into $H$; hence we propose the following:

Definition. A semiclosed operator in $H$ is an operator in $H$ which, as a subspace of $H \times H$, is semiclosed.

Evidently each closed operator in $H$ is semiclosed, since any closed subspace is semiclosed. However, there exists a semiclosed operator in $\boldsymbol{H}$ whose closure is all of $H \times H$ (see §2). In fact, every function from a countable linearly independent subset of $H$ into $H$ has an extension which is a semiclosed linear operator in $H$ (Theorem 12).

Herein, we show that the family $\mathcal{S}(H)$ of all semiclosed operators in $H$ is closed under addition, multiplication, inversion, restriction to semiclosed subspaces of $H$, and strong limits in $H$ on such subspaces. Moreover, we find each of the following to be a necessary and sufficient condition on an operator $C$ in $H$ in order that $C$ be in $\mathcal{S}(H)$ (let $S$ denote the domain of C):

(I) $C$ is the sum of two closed reversible (one-to-one) operators in $H$ each having domain $S$ and closed range (hence, continuous inverses).

(II) $C$ is the product of a continuous operator on $H$ with a closed positive

Presented to the Society, January 24, 1979; received by the editors September 8, 1978.

AMS (MOS) subject classifications (1970). Primary 47A45, 47A65.

Key words and phrases. Closed operator, semiclosed operator, Hilbert space, complete inner product space, operator ranges. 
operator in $H$ having domain $S$ and closed range.

(III) $C$ is an algebraic combination of closed operators in $H$.

(IV) $S$ is a semiclosed subspace of $H$ and $C$ is the strong limit in $H$ on $S$ of a sequence of continuous operators on $H$.

(V) $S$ is a semiclosed subspace of $H$ and $C$ is continuous from $S$ with its natural topology into $H$.

From these facts, it is easy to see that the semiclosed operators in $H$ constitute the smallest family of operators in $H$ which contains the closed operators and is itself closed under either addition, multiplication, or strong limits on semiclosed subspaces

The phrase "natural topology" in (V) arises from the fact that if $S$ is a semiclosed subspace of $H$ then all inner products $\langle\cdot, \cdot\rangle^{\prime}$ as in the definition generate the same topology on $S$ (see Lemma $\mathrm{A}$ below and the comments following). Accordingly, we regard each such subspace $S$ as equipped with this topology, and for each pair $\left(S, S^{\prime}\right)$ of such subspaces, we denote by $\mathscr{B}\left(S, S^{\prime}\right)$ the family of all operators in $H$ continuous from $S$ to $S^{\prime}$, and abbreviate $\mathscr{B}(S, S)$ to $\mathscr{B}(S)$. In particular, $\mathscr{B}(H)$ denotes the usual family of all continuous (i.e., bounded) operators from $\boldsymbol{H}$ to $\boldsymbol{H}$. Norms corresponding to various inner products $\langle\cdot, \cdot\rangle,\langle\cdot, \cdot\rangle \sim,\langle\cdot, \cdot\rangle_{0}$, etc. will be denoted by $\|\cdot\|,\|\cdot\|^{\sim},\|\cdot\|_{0}$, etc., except that if $A$ is in $\mathscr{B}(H)$ then $\|A\|$ denotes the operator norm of $A$ in $(H,\langle\cdot, \cdot\rangle)$. The following convenient construction will also be used throughout: if $A$ is in $\mathscr{B}(H)$ then $A^{-1}$ denotes the inverse of the restriction of $A$ to the orthogonal complement of the null space of $A$. Note that $A^{-1}$ maps $A(H)$ onto the closure of $A^{*}(H)$ and $A^{-1} A$ is the orthogonal projection from $H$ onto said closure.

We take for granted the basic facts about semiclosed subspaces as compiled by Fillmore and Williams in [1], e.g. that the set of all of them is the lattice of domains of closed operators in $H$, and include the following compendium of results of Mac Nerney [3, Theorem 3, p. 666], and [4, p. 110], upon which a number of our proofs are based:

LeMma A. A subspace $S$ of $H$ is semiclosed in $H$ only in case $S$ is the range of a member of $\Re(H)$. In this case, for each inner product $\langle\cdot, \cdot\rangle^{\prime}$ for $S$ such that $\left(S,\langle\cdot, \cdot\rangle^{\prime}\right)$ is complete and continuously included in $H$, there is only one nonnegative member $A$ of $\mathscr{B}(H)$ such that $A(H) \subset S$ and $\langle x, A z\rangle^{\prime}=\langle x, z\rangle$ for all $(x, z)$ in $S \times H$. Moreover, if $B$ is in $\Re(H)$ and $B B^{*}=A$ then $B(H)=S$ and $\langle x, y\rangle^{\prime}=\left\langle B^{-1} x, B^{-1} y\right\rangle$ for all $(x, y)$ in $S \times S$.

It may be shown with the aid of Lemma $A$ that if $S$ and $S^{\prime}$ are semiclosed subspaces of $H$ and $S \subset S^{\prime}$ then $S$ is continuously included in $S^{\prime}$; thus the notion of a semiclosed subspace is, in a certain sense, not dependent upon the choice of the inner product therein or the containing space. 


\section{Initial characterization and elementary operations.}

THEOREM 1. If $C$ is an operator in $H$ then the following are equivalent:

(1) $C$ is in $\mathrm{S} \mathcal{C}(H)$;

(2) the domain of $C$ is a semiclosed subspace $S$ of $H$ such that $C$ belongs to $\mathscr{B}(S, H)$;

(3) there is a member $(A, B)$ of $\mathscr{B}(H) \times \mathscr{B}(H)$ such that $C$ is the quotient $A B^{-1}$; and

(4) there is such a pair $(A, B)$ as in (3) with the property that $B$ is nonnegative and $A\left(B^{-1} B\right)=A$.

Proof. Let $S$ denote the domain of $C$. Suppose (1) is true, and let $\langle\cdot, \cdot\rangle_{0}$ denote an inner product for $C$ such that $\left(C,\langle\cdot, \cdot\rangle_{0}\right)$ is complete and continuously included in $H \times H$. By definition, there is a nonnegative number $b$ such that for all $x$ in $S,\|(x, C x)\|^{\sim} \leqslant b\|(x, C x)\|_{0}$. Now for each $(x, y)$ in $S \times S$, let $\langle x, y\rangle_{1}=\langle(x, C x),(y, C y)\rangle_{0}$ : clearly $\langle\cdot, \cdot\rangle_{1}$ is an inner product for $S$ such that $\left(S,\langle\cdot, \cdot\rangle_{1}\right)$ is complete, and for each $x$ in $S$, $\|x\|^{2}+\|C x\|^{2} \leqslant b^{2}\|x\|_{1}^{2}$. This establishes that (2) is true.

We now prove that (2) implies (4). Suppose (2), and let $\langle\cdot, \cdot\rangle_{1}$ denote an inner product for the semiclosed subspace $S$ of $H$ as in the definition. By Lemma $A$, there is a nonnegative member $B$ of $\mathscr{B}(H)$ such that $B(H)=S$ and, for all $(x, y)$ in $S \times S,\langle x, y\rangle_{1}=\left\langle B^{-1} x, B^{-1} y\right\rangle$. Let $A=C B: B$ is certainly in $\Re(H, S)$ and $C$ is by hypothesis in $\mathscr{B}(S, H)$, so $A$ is in $\mathscr{B}(H)$. Since $B B^{-1}$ is simply the identity function on $S, A B^{-1}=C B B^{-1}=C$ and $A B^{-1} B=C B=A$. Hence (4) is true.

Since (4) is just a special case of (3), it remains only to show that (3) implies (1). Suppose, therefore, that (3) holds true, and for all $(x, y)$ in $H \times H$, let $F(x, y)=(B x, C B x)$. It is easy to see that $F$ maps $H \times H$ onto $C$, since $C$ has domain $B(H)$. Also, $F$ is linear, and if $m$ denotes $\sup \{\|A\|,\|B\|\}$, then for all $(x, y)$ in $H \times H$, we have that

$$
\|F(x, y)\|^{2}=\|B x\|^{2}+\left\|A B^{-1} B x\right\|^{2}<2 m^{2}\|x\|^{2}<2 m^{2}\|(x, y)\|^{2} .
$$

Thus $F$ is in $\mathscr{B}(H \times H)$, so via the application of Lemma $\mathrm{A}$ in $H \times H$ rather than $H$, we see that the range of $F$ is a semiclosed subspace of $H \times H$. Thus (1) is true, and the proof is complete.

REMARKS. Statements (2) and (4) of Theorem 1 correspond to characterizations (V) and (II) of the Introduction, respectively. Note also that (II) implies that each member of $\mathcal{S}(H)$ with closed domain is continuous; this is a special case of the "semiclosed graph theorem" (see [1, p. 258]).

Theorem 2. Suppose that $C$ is in $\mathcal{S}(H), S$ is the domain of $C$, and $S^{\prime}$ is a semiclosed subspace of $H$. Then each of these holds true:

(i) each of $S$ and $C(S)$ is a semiclosed subspace of $H$;

(ii) if $S^{\prime} \subset S$ then the restriction of $C$ to $S^{\prime}$ is in $\delta \mathcal{C}(H)$;

(iii) if $S^{\prime} \subset S$ then $C\left(S^{\prime}\right)$ is a semiclosed subspace of $H$; 
(iv) if $S^{\prime} \subset C(S)$ then $C^{-1}\left(S^{\prime}\right)$ is a semiclosed subspace of $H$;

(v) if $C$ is reversible then $C^{-1}$ is in $\mathcal{S} \mathcal{C}(H)$.

Proof. Statement (i) follows at once from (3) of Theorem 1 and the fact that each member of $\mathscr{B}(H)$ has range a semiclosed subspace of $H$. Suppose that $S^{\prime} \subset S$ and let $I$ denote the identity function on $S^{\prime}$. By the comment following Lemma $\mathrm{A}, I$ is in $\mathscr{B}\left(S^{\prime}, S\right)$, and by (2) of Theorem $1, C$ is in $\Re(S, H)$. Hence $C I$ is in $\Re\left(S^{\prime}, H\right)$ and therefore in $\mathcal{S}(H)$. But $C I$ is just the restriction of $C$ to $S^{\prime}$; hence (ii) is true. Moreover, by (i) $C I\left(S^{\prime}\right)=C\left(S^{\prime}\right)$ is a semiclosed subspace of $H$-so (iii) is also true.

Now suppose that $S^{\prime} \subset C(S)$ and let $D=C \cap\left(H \times S^{\prime}\right)$. Since $C$ and $H \times S^{\prime}$ are both semiclosed subspaces of $H, D$ is also. However, $D$ is a subset of $C$ and therefore an operator in $H$; hence $D$ is in $\delta \mathcal{C}(H)$. Since $D$ has domain $C^{-1}\left(S^{\prime}\right)$, (iv) is proved by applying (i) to $D$.

Finally, suppose $C$ is reversible and let $F(x, y)=(y, x)$ for all $(x, y)$ in $H \times H$. Then $F$ is a semiclosed (in fact, continuous) operator in $H \times H$ which maps $C$ onto $C^{-1}$; hence by applying (iii) to $F$ and $C$ rather than $C$ and $S^{\prime}$, we get $(v)$, and all is proved.

THEOREM 3. If each of $C_{1}$ and $C_{2}$ is in $\mathcal{S}(H)$ then each of $C_{1}+C_{2}$ and $C_{1} C_{2}$ is also.

Proof. Let $S_{1}$ and $S_{2}$ denote the domains of $C_{1}$ and $C_{2}$, respectively: $S_{1} \cap S_{2}$ is a semiclosed subspace of $H$ (since by (i) of Theorem 2, each of $S_{1}$ and $S_{2}$ is), and thus by (ii) of Theorem 2 the restrictions of $C_{1}$ and $C_{2}$ thereto are in $\mathcal{S}(H)$. By Theorem 1, each of these restrictions is in the linear space $\mathscr{B}\left(S_{1} \cap S_{2}, H\right)$. Thus $C_{1}+C_{2}$ is in $\mathscr{B}\left(S_{1} \cap S_{2}, H\right)$ and therefore-again by Theorem 1-in $\mathcal{S}(\boldsymbol{H})$.

Now let $S_{3}$ denote $S_{1} \cap C_{2}\left(S_{2}\right)$. By (iv) of Theorem $2, C^{-1}\left(S_{3}\right)$ is a semiclosed subspace of $H$ : let $C_{3}$ denote the restriction of $C_{2}$ thereto. Then $C_{1} C_{2}=C_{1} C_{3}$, with $C_{1}$ in $\mathscr{B}\left(S_{1}, H\right)$ and $C_{3}$ in $\mathscr{B}\left(C_{2}^{-1}\left(S_{3}\right), S_{1}\right)$. It now follows that $C_{1} C_{2}$ is in $\Re\left(C_{2}^{-1}\left(S_{3}\right), H\right)$ and hence in $\mathcal{S}(H)$.

REMARK. Theorem 3 may be used to show that each member $C$ of $\mathcal{S}(H)$ has an extension in $\mathcal{S}(H)$ with domain dense in $H$ (let $C^{\prime}=C P$, where $P$ is the projection from $H$ onto the closure of the domain of $C$ ).

TheOREM 4. Suppose that $S$ is a semiclosed subspace of $H, \gamma$ is an infinite sequence each term of which is a member of $\mathcal{S}(H)$ having domain including $S$, and $C$ is the strong limit in $H$ of $\gamma$ on $S$. Then $C$ is in $\mathcal{S}(H)$.

Proof. For each nonnegative integer $n$, the restriction of $\gamma_{n}$ to $S$ is in $\mathcal{S} \mathcal{C}(H)$ (by Theorem 2) and therefore in $\mathscr{B}(S, H)$ (by Theorem 1). That $C$ is in $\mathscr{B}(S, H)$ and hence in $\mathcal{S}(H)$ is an immediate consequence of the uniform boundedness principle. 
THEOREM 5. Characterization (IV) of the introduction is a necessary and sufficient condition on an operator $C$ in $H$ with domain $S$ in order that $C$ be in $\mathcal{S}(H)$.

Proof. That (IV) is sufficient is easily seen by taking $\gamma$ of Theorem 4 to be $\mathscr{B}(H)$-valued. To prove the necessity, we let $C=A B^{-1}$ as in (4) of Theorem 1. Since $B$ is nonnegative, $B^{-1}$ is a selfadjoint operator in the space $(M$, $\langle\cdot, \cdot\rangle)$, where $M$ denotes the closure of $B(H)$ in $H$. Let $\phi$ denote the spectral resolution of $B^{-1}$ in that space, let $P$ denote the projection from $H$ onto $M$, and for each nonnegative integer $n$ let $\gamma_{n}=A \int_{0}^{n} I d \phi P$, where $I$ denotes the real identity function. Since $S$ lies in $M$, it now follows that $C$ is the strong limit in $H$ on $S$ of the sequence $\gamma$ so defined, and this completes the proof.

\section{Closed operators and the quotient representation.}

THEOREM 6. suppose that $(A, B)$ is in $\mathscr{B}(H) \times \mathscr{B}(H), A\left(B^{-1} B\right)=A$, and $C=A B^{-1}$. Then $C$ is a closed operator in $H$ only in case the vector sum $A^{*}(H)+B^{*}(H)$ is a closed subspace of $H$.

INDICATION OF PROOF. This is essentially proved as Theorem 1 of [2], in which an operator $C$ is shown to be closed only in case there exists a quotient $A B^{-1}$ such that $A^{*}(H)+B^{*}(H)$ is closed- without the additional requirement that $A\left(B^{-1} B\right)=A$. The main idea is the identification of the function $J$ defined by $J(z)=\left(B D^{-1} z, A D^{-1} z\right)$ for all $z$ in $D(H)$, where $D=\left(A^{*} A+\right.$ $\left.B^{*} B\right)^{1 / 2}$, as a linear isometry from $\left(A^{*}(H)+B^{*}(H),\langle\cdot, \cdot\rangle\right)$ onto $\left(A B^{-1}\right.$, $\left.\langle\cdot, \cdot\rangle^{\sim}\right)$, from which fact the stated equivalence is obvious.

TheOREM 7. If $(A, B)$ is in $\mathscr{B}(H) \times \mathscr{B}(H)$ and $A$ is invertible then $A B^{-1}$ is a closed operator in $H$.

Proof. Let $A^{\prime}=A\left(B^{-1} B\right)$. Then $A^{\prime *}(H)+B^{*}(H)=B^{-1} B A^{*}(H)+$ $B^{*}(H)=B^{-1} B(H)$ (the last equality holds since $A^{*}(H)=H$ and $B^{-1} B(H)$ is the closure of $B^{*}(H)$ ). Hence $A^{*}(H)+B^{*}(H)$ is closed in $H$, so that by Theorem 6, $A B^{-1}\left(=A^{\prime} B^{-1}\right)$ is a closed operator in $H$.

THEOREM 8. If $C$ is in $\mathcal{S}(H)$ then there is a member $(F, G)$ of $\mathfrak{B}(H) \times$ $\mathscr{B}(H)$ such that each of $F$ and $G$ has range the domain of $C$, and $C=F^{-1}+$ $G^{-1}$.

Proof. Let $C=A B^{-1}$ as in (4) of Theorem 1, and let $\lambda$ denote any complex number in the resolvent set of $A$ (e.g., $\|A\|+1)$ : since $A-\lambda$ is invertible, it follows from Theorem 7 that $(A-\lambda) B^{-1}$ is a closed operator $E$ in $H$. Moreover, the range of $E$ is the closed subspace $E B(H)$ of $H$, which implies that $E^{-1}$ is continuous in $H$. Let $P$ denote the projection from $H$ onto $E B(H)$, let $F$ denote the extension $E^{-1} P$ of $E^{-1}$ to $H$, and let $G$ denote $\lambda^{-1} B$. Then $F^{-1}=(A-\lambda) B^{-1}$ and $G^{-1}=\lambda B^{-1}$, so that $F(H)=G(H)=$ $B(H)$ and $F^{-1}+G^{-1}=(A-\lambda+\lambda) B^{-1}=C$, as was to be proved. 
THEOREM 9. Characterization (I) of the introduction is a necessary and sufficient condition on an operator $C$ in $H$ with domain $S$ in order that $C$ be in $\mathcal{S}(\boldsymbol{H})$.

Proof. This is a corollary to Theorems 3 and 8.

Example. There exists a pair $(A, B)$ of positive members of $\mathscr{B}(H)$ (i.e., $\langle x, A x\rangle>0$ and $\langle x, B x\rangle>0$ unless $x=0)$ with $A(H)$ and $B(H)$ having only the point 0 in common [1, Theorem 3.6, p. 273]. Let $C=A B^{-1}$ and suppose that $(x, y)$ is in the orthogonal complement of $C$ in $H \times H$. Then for all $z$ in $H$,

$$
\langle B x+A y, z\rangle=\langle x, B z\rangle+\langle y, A z\rangle=\langle(x, y),(B z, C B z)\rangle^{\sim}=0 .
$$

Hence $B x+A y=0$ and therefore $x=y=0$. Thus $C$ has closure $H \times H$. Decomposition of $C$ as in Theorem 8 provides us with a pair of closed operators in $H$ whose sum is dense in $H \times H$.

\section{Semiclosed extensions.}

Definition. If each of $D$ and $E$ is an operator in $H$ then $D$ is continuous with respect to $E$ provided that the domain of $E$ includes that of $D$ and there is a nonnegative number $b$ such that $\|D x\| \leqslant b\|E x\|$ for all $x$ in the domain of $D$.

THEOREM 10. If $D$ is an operator in $H$ then each of the following is a necessary and sufficient condition in order that $D$ have an extension in $\mathcal{S}(H)$ :

(1) there is a member $B$ of $\mathscr{B}(H)$ such that $D$ is continuous with respect to $B^{-1}$,

(2) $D$ is continuous with respect to a selfadjoint operator in $H$,

(3) $D$ is continuous with respect to a closed operator in $H$, and

(4) $D$ is continuous with respect to a semiclosed operator in $H$.

Proof. We show first that the four numbered statements are equivalent. Suppose (1) is true, and let $C$ denote the extension $B^{-1}\left(B^{*-1} B^{*}\right)$ of $B^{-1}$ (see the remark following Theorem 3). Since $C$ is in $\mathcal{S}(H)$, there is a member $(A, G)$ of $\mathscr{B}(H) \times \mathscr{B}(H)$ such that $C=A G^{-1}$ as in (4) of Theorem 1, and since $C$ has domain dense in $H$, the operator $G$ is positive. Hence $G^{-1}$ is selfadjoint. Now by hypothesis, there is a nonnegative number $b$ such that for all $x$ in the domain of $D,\|D x\| \leqslant b\left\|B^{-1} x\right\|$ and thus $\|D x\|<b\|C x\|<$ $b\|A\|\left\|G^{-1} x\right\|$. Hence (2). That (2) implies (3), and that (3) implies (4), is obvious, so suppose (4). Representing the hypothesized semiclosed operator as a quotient $A B^{-1}$ as usual, we have that for some nonnegative number $b$, $\|D x\|<b\left\|A B^{-1} x\right\| \leqslant b\|A\|\left\|B^{-1} x\right\|$ for all $x$ in the domain of $D$, so that (1) is true.

Now suppose that $D$ has a semiclosed extension in $\boldsymbol{H}$. Since this is just the special case of the immediately preceding argument that (4) implies (1) in which $D x=A B^{-1} x$, we get (1). Finally, suppose once again that (4) holds true, with $b$ and $A B^{-1}$ as before. For each $z$ in $H$ such that $B z$ is in the 
domain of $D,\|D B z\| \leqslant b\|A\|\left\|B^{-1} B z\right\| \leqslant b\|A\|\|z\|$. Thus $D B$ has an extension $E$ which belongs to $\mathscr{B}(H)$, and for any such $E$ and $x$ in the domain of $D, D x=D B B^{-1} x=E B^{-1} x$-so that $E B^{-1}$ is a semiclosed extension of $D$. This completes our proof.

TheOREM 11. Suppose that $D$ is an operator in $H$ with domain $S$ and that there exists a monotonic collection $J$ of closed subspaces of $H$ such that $J$ covers $S$ and for each $M$ in $J$, the restriction of $D$ to $M \cap S$ is continuous in $H$. Then $D$ has a semiclosed extension in $H$.

Proof. Let $\beta$ denote the function on $J$ such that, for each $M$ in $J, \beta(M)$ is the least nonnegative number $b$ such that $\|D x\|<b\|x\|$ for all $x$ in $M \cap S$. If $\beta$ is bounded then $D$ is continuous in $H$, so suppose not and let $\left\{M_{n}\right\}_{0}^{\infty}$ denote an increasing sequence of members of $J$ such that $\left\{\beta\left(M_{n}\right)\right\}_{0}^{\infty}$ is unbounded. Let $K$ denote the orthogonal complement of $\cup J$ in $H$, and for each nonnegative integer $n$, let $P_{n}$ denote the projection from $H$ onto $M_{n}+K$. Now let $P_{-1}=0$ and let $C=\sum_{n=0}^{\infty} \beta\left(M_{n}\right)\left(P_{n}-P_{n-1}\right)$.

Since $\beta\left(M_{n}\right)$ is real nonnegative for each $n$, it follows that $C$ is a selfadjoint operator in $H$, and since $S$ is covered by the set of all terms of $\left\{P_{n}(H)\right\}_{0}^{\infty}$, we have that $D$ is continuous with respect to $C$ in the sense indicated at the beginning of this section. Thus by (2) of Theorem 10, $D$ has an extension in $\mathcal{S}(H)$.

THEOREM 12. Each function from a countable linearly independent subset of $\boldsymbol{H}$ into $H$ has an extension in $\mathcal{S}(H)$.

Proof. This is a consequence of Theorem 11 via the observation that the linear span of such a subset is the union of a monotonically increasing sequence of finite-dimensional subspaces.

ADDED IN PROOF. It has lately been brought to the author's attention that there are some points of contact between this work and [5, esp. pp. 37-50], in which the single-valued J-operators are recognizable as the objects which are termed "semiclosed operators" herein.

\section{REFERENCES}

1. P. A. Fillmore and J. P. Williams, On operator ranges, Advances in Math. 7 (1971), 254-281.

2. W. E. Kaufman, Representing a closed operator as a quotient of continuous operators, Proc. Amer. Math. Soc. 72 (1978), 531-534.

3. J. S. Mac Nearney, Investigation concerning positive definite continued fractions, Duke Math. J. 26 (1959), 663-678.

4. , Continuous embeddings of Hilbert spaces, Rend. Circ. Mat. Palmero (2) 19 (1970), $109-112$.

5. J. Dixmier, Étude sur les variétés et les opérateurs de Julia, Bull. Soc. Math. France 77 (1949), 11-101.

Department of Mathematics, University of Houston, Houston, Texas 77004 